\title{
Bright Soliton Solution of $(1+1)$-Dimensional Quantum System with Power-Law Dependent Nonlinearity
}

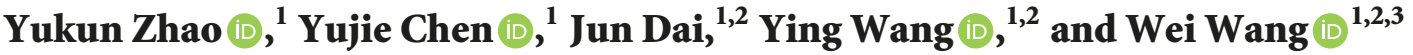 \\ ${ }^{1}$ School of Science, Jiangsu University of Science and Technology, Zhenjiang 212003, China \\ ${ }^{2}$ Laboratory of Advanced Optics, Jiangsu University of Science and Technology, Zhenjiang 212003, China \\ ${ }^{3}$ Institute of Photonics and Quantum Sciences, School of Engineering and Physical Sciences, Heriot-Watt University Edinburgh, \\ EH14 4AS, UK
}

Correspondence should be addressed to Ying Wang; wangying@just.edu.cn and Wei Wang; w.wang@hw.ac.uk

Received 5 January 2019; Accepted 27 January 2019; Published 3 March 2019

Academic Editor: Shuo Yin

Copyright (C) 2019 Yukun Zhao et al. This is an open access article distributed under the Creative Commons Attribution License, which permits unrestricted use, distribution, and reproduction in any medium, provided the original work is properly cited.

We study the nonlinear dynamics of $(1+1)$-dimensional quantum system in power-law dependent media based on the nonlinear Schrödinger equation (NLSE) incorporating power-law dependent nonlinearity, linear attenuation, self-steepening terms, and third-order dispersion term. The analytical bright soliton solution of this NLSE is derived via the F-expansion method. The key feature of the bright soliton solution is pictorially demonstrated, which together with typical analytical formulation of the soliton solution shows the applicability of our theoretical treatment.

\section{Introduction}

In the fascinating study of physical reality, nonlinear phenomena have attracted significant attention. Besides experimental observation of nonlinear behaviors, for the theoretical investigation of nonlinearity, the nonlinear Schrödinger equation (NLSE) has proved to be a reliable model under certain experimental settings because it is closely related to many nonlinear problems and people have conducted extensive research on finding solutions [1-15] of particular nonlinear features. For a practical theoretical treatment, the one-dimensional scenario of the NLSE model is typical not only because of the integrability of the system under many parametric settings, but also because that actual experimental observation identifies stable soliton behavior under such a scenario. Therefore, the $(1+1)$-dimensional case of the NLSE is the focus of many theoretical works, including this study.

For the analytical study of various categories of the $(1+1)$ dimensional NLSE, many approaches have been proposed, such as the Bäcklund transformation method [16], homotopy analysis method [17], variational iteration method, and Expfunction method [2]. For a typical analytical solution investigation of the NLSE in this study, we utilize the F-expansion method, which features the Jacobian elliptic function expansion to solve NLSE for typical traveling wave solutions. It is a very effective method to solve partial differential equations such as NLSE. Basically, it is implemented by expressing the solutions of the equations in the form of power expansion of Jacobian function [18, 19]. Moreover, for the nonlinear study of the NLSE, the soliton-type solution is usually the ultimate goal of the analytical solution search. This usually comes from the desirable features of soliton. Because it is wave solution of a nonlinear wave equation, it can propagate for a long distance without deformation, and its amplitude, shape, and speed remain unchanged when it meets other similar solitary waves. In this paper, we study the soliton behavior of the NLSE by modeling the nonlinear dynamics in power-law dependent media such as an optical fiber system, where the group velocity dispersion term and nonlinear term are present and adjustable. The linear attenuation term, selfsteepening term, and third-order dispersion term also exist [20]. We identify that, under an appropriate experimental setting, the bright soliton solutions can be derived via the $F$ expansion method, and the typical bright soliton feature is pictorially demonstrated.

This paper is organized as follows. The next section gives the NLSE model formulation of this study, Section 3 describes 
the procedural detail of the $F$-expansion method, Section 4 gives the concrete calculation steps of solving the NLSE for its solition solution, and the last section gives the conclusive remarks.

\section{Model and Method}

2.1. NLSE Model in Power-Law Dependent Media. For studying the soliton dynamics in a $(1+1)$-dimensional power-law dependent media $[2,10,15,20]$, the NLSE in dimensionless format is

$$
\begin{aligned}
i \psi_{t} & +a(t) \psi_{x x}+b(t)|\psi|^{2 m} \psi \\
& =i \alpha(t)] \psi+i \lambda(t)\left(|\psi|^{2 m} \psi\right)_{x}-i \gamma(t) \psi_{x x x}
\end{aligned}
$$

The independent variables in $\psi$ include the space $x$ and time $t$ coordinates, $i \psi_{t}$ describes the evolution of wave function, $a(t) \psi_{x x}$ is the term of group velocity dispersion, $b(t)|\psi|^{2 m} \psi$ is the nonlinear term, $a(t)$ and $b(t)$ are the real valued functions of time $t, \alpha(t)$ is the time-dependent coefficient of linear attenuation term, $\lambda(t)$ is the time-dependent coefficient of self-steepening term, and $\gamma(t)$ is the time-dependent coefficient of the third-order dispersion term. Here, we consider the case where the coefficient parametric functions of various terms in (1) do not vary with time within a certain period but can be freely adjusted according to the experimental setting.

2.2. The F-Expansion Method. The F-expansion method is used to solve general partial differential equations of the following form:

$$
G\left(u, u_{t}, u_{x}, u_{x x}, \ldots\right)=0
$$

where $u(x, t)$ is the unknown function to be solved and $G$ is the polynomial of $u(x, t)$ and its partial derivatives of various orders. The F-expansion method is implemented through the use of the polynomial of base function $F(\xi)$ to express $u(x, t)$, where $F(\xi)$ is defined as

$$
\begin{aligned}
\left(\frac{d F(\xi)}{d \xi}\right)^{2}= & F(\xi)^{4}+b_{3} F(\xi)^{3}+b_{2} F(\xi)^{2}+b_{1} F(\xi) \\
& +b_{0}
\end{aligned}
$$

where

$$
\xi=p x+q t
$$

Here $p, q, b_{0}, b_{1}, b_{2}, b_{3}$ are parametric constants to be determined in the problem-solving steps. $u(x, t)$ is expressed through $F(\xi)$ and it takes the following form:

$$
u(x, t)=\sum_{i=0}^{m} h_{i}(t) F^{i}(\xi)
$$

where the highest order number $m$ is determined by balancing the highest order of nonlinearity and highest order of derivatives. By inserting (5) into (2) and using the definition of $F(\xi)$, we obtain a polynomial of $F(\xi)$. After setting the coefficients of various terms of the polynomial, we obtain a set of ordinary differential equations (ODEs) of time $t$. Solving the ODEs consistently, we obtain the analytical expressions of the parametric constants in (3) and $h_{i}(t)$ in (5), and (2) is solved accordingly.

\section{Procedural Details and Results for Reaching Solition Solution of NLSE in Power-Law Dependent Media}

To solve (1), we can assume that $\psi(x, t)$ takes the following traveling wave format:

$$
\psi(x, t)=\varphi(\xi) e^{i(A x+B t)}
$$

Substituting (6) into (1), we get

$$
\begin{aligned}
i \psi_{t} & =\left(i q \varphi^{\prime}-A \varphi\right) e^{i(A t+B x)} \\
\psi_{x} & =\left(\varphi^{\prime} p+i B \varphi\right) e^{i(A t+B x)} \\
\psi_{x x} & =\left(\varphi^{\prime \prime} p^{2}+2 i B \varphi^{\prime} p-B^{2} \varphi\right) e^{i(A t+B x)} \\
\psi_{x x x} & =\left(\varphi^{\prime \prime \prime} p^{3}+3 i B \varphi^{\prime \prime} p^{2}-3 B^{2} \varphi^{\prime} p-i B^{3} \varphi\right) e^{i(A t+B x)}
\end{aligned}
$$

Plugging (7a), (7b), (7c), and (7d) into (1), we get

$$
\begin{aligned}
\left(i q \varphi^{\prime}\right. & -A \varphi)+a(t)\left(\varphi^{\prime \prime} p^{2}+2 i B \varphi^{\prime} p-B^{2} \varphi\right) \\
& +b(t) \varphi^{2 m+1} \\
= & i \alpha(t) \varphi+i \lambda(t)(2 m+1) \varphi^{2 m} \varphi^{\prime} p \\
& -\lambda(t)(2 m+1) B \varphi^{2 m+1}+i \gamma(t) \varphi^{\prime \prime \prime} p^{3} \\
& -3 B \gamma(t) \varphi^{\prime \prime} p^{2}-3 i B^{2} \gamma(t) \varphi^{\prime} p+B^{3} \gamma(t) \varphi
\end{aligned}
$$

The real part of (8) is

$$
\begin{aligned}
{[b(t)} & +\lambda(t)(2 m+1) B] \varphi^{2 m+1} \\
& -\left[A+a(t) B^{2}+\gamma(t) B^{3}\right] \varphi \\
& +\left[a(t) p^{2}+3 \gamma(t) B p^{2}\right] \varphi^{\prime \prime}=0
\end{aligned}
$$

The imaginary part of (8) is

$$
\begin{aligned}
\lambda(t) & (2 m+1) p \varphi^{2 m} \varphi^{\prime}+\alpha(t) \varphi \\
& -\left[q+2 a(t) B p+3 \gamma(t) B^{2} p\right] \varphi^{\prime}+\gamma(t) p^{3} \varphi^{\prime \prime \prime} \\
& =0
\end{aligned}
$$

We define

$$
T(\varphi)=\left[\frac{d \varphi(\xi)}{d \xi}\right]^{2}=\left(\varphi^{\prime}\right)^{2}
$$

So

$$
\frac{d T}{d \xi}=\frac{d T}{d \varphi} \frac{d \varphi}{d \xi}=\varphi^{\prime} \frac{d T}{d \varphi}
$$


and

$$
\varphi^{\prime \prime}=\frac{d \varphi^{\prime}}{d \xi}=\varphi^{\prime} \frac{d \varphi^{\prime}}{d \varphi}=\frac{1}{2} \frac{d \varphi^{\prime 2}}{d \varphi}=\frac{1}{2} \frac{d T}{d \varphi}=\frac{1}{2} T^{\prime}
$$

Substituting (13) into (9), we obtain

$$
\lambda_{1} \varphi^{2 m+1}-\lambda_{2} \varphi+\frac{1}{2} \lambda_{3} T^{\prime}=0
$$

where

$$
\begin{aligned}
& \lambda_{1}=b(t)+\lambda(t)(2 m+1) B \\
& \lambda_{2}=A+a(t) B^{2}+\gamma(t) B^{3} \\
& \lambda_{3}=a(t) p^{2}+3 \gamma(t) B p^{2}
\end{aligned}
$$

For the implementation of the $F$-expansion method, we set $\varphi(\xi)=F(\xi)$, and the $F$ base function is chosen as follows:

$$
T=a_{m} \varphi^{2 m+2}+a_{2} \varphi^{2}
$$

Plugging (16) into (14), and performing integration, we get the coefficients as follows:

$$
\begin{aligned}
\varphi^{2 m+1}: \lambda_{1}+(m+1) \lambda_{3} a_{m} & =0 \\
\varphi:-\lambda_{2}+\lambda_{3} a_{2} & =0
\end{aligned}
$$

From (17a) and (17b), we get

$$
\begin{aligned}
& a_{2}=\frac{\lambda_{2}}{\lambda_{3}} \\
& a_{m}=-\frac{\lambda_{1}}{(m+1) \lambda_{3}}
\end{aligned}
$$

Plugging (15a), (15b), and (15c) into (18a) and (18b), we get

$$
\begin{aligned}
a_{2} & =\frac{A+a(t) B^{2}+\gamma(t) B^{3}}{a(t) p^{2}+3 \gamma(t) B p^{2}} \\
a_{m} & =-\frac{b(t)+\lambda(t)(2 m+1) B}{(m+1)\left[a(t) p^{2}+3 \gamma(t) B p^{2}\right.}
\end{aligned}
$$

We get $\varphi^{\prime \prime}$ from (9):

$$
\varphi^{\prime \prime}=-\frac{\lambda_{1}}{\lambda_{3}} \varphi^{2 m+1}+\frac{\lambda_{2}}{\lambda_{3}} \varphi
$$

We differentiate both sides of (20):

$$
\varphi^{\prime \prime \prime}=\frac{d \varphi^{\prime \prime}}{d \xi}=-\frac{\lambda_{1}}{\lambda_{3}}(2 m+1) \varphi^{2 m} \varphi^{\prime}+\frac{\lambda_{2}}{\lambda_{3}} \varphi^{\prime}
$$

Equation (8) then takes the following form:

$$
\lambda_{4} \varphi^{2 m} \varphi^{\prime}+\alpha(t) \varphi-\lambda_{5} \varphi^{\prime}+\lambda_{6} \varphi^{\prime \prime \prime}=0
$$

Here

$$
\begin{aligned}
& \lambda_{4}=\lambda(t)(2 m+1) p \\
& \lambda_{5}=q+2 a(t) B p+3 \gamma(t) B^{2} p \\
& \lambda_{6}=\gamma(t) p^{3}
\end{aligned}
$$

Plugging (21) into (22), we get

$$
\begin{gathered}
{\left[\lambda_{4}-\lambda_{6} \frac{\lambda_{1}}{\lambda_{3}}(2 m+1)\right] \varphi^{2 m} \varphi^{\prime}+\alpha(t) \varphi} \\
+\left[\lambda_{6} \frac{\lambda_{2}}{\lambda_{3}}-\lambda_{5}\right] \varphi^{\prime}=0
\end{gathered}
$$

Dividing both sides of (24) by $\varphi$, and then taking both derivatives with respect to $\xi$, we get

$$
\begin{aligned}
{\left[\lambda_{4}\right.} & \left.-\lambda_{6} \frac{\lambda_{1}}{\lambda_{3}}(2 m+1)\right](2 m-1) \varphi^{2 m-2} \varphi^{\prime 2} \\
& +\left[\lambda_{4}-\lambda_{6} \frac{\lambda_{1}}{\lambda_{3}}(2 m+1)\right] \varphi^{2 m-1} \varphi^{\prime \prime} \\
& +\left[\lambda_{6} \frac{\lambda_{2}}{\lambda_{3}}-\lambda_{5}\right] \frac{\varphi^{\prime \prime}-\varphi^{\prime 2}}{\varphi^{2}}=0
\end{aligned}
$$

Substituting (11) and (13) into (25), we get

$$
\begin{aligned}
{\left[\lambda_{4}\right.} & \left.-\lambda_{6} \frac{\lambda_{1}}{\lambda_{3}}(2 m+1)\right](2 m-1) \varphi^{2 m-2} T \\
& +\left[\lambda_{4}-\lambda_{6} \frac{\lambda_{1}}{\lambda_{3}}(2 m+1)\right] \varphi^{2 m-1} \frac{1}{2} T^{\prime} \\
& +\left[\lambda_{6} \frac{\lambda_{2}}{\lambda_{3}}-\lambda_{5}\right] \frac{(1 / 2) T^{\prime}-T}{\varphi^{2}}=0
\end{aligned}
$$

Plugging (16) into (26), performing the integration, we get the coefficient of each term as

$$
\begin{aligned}
& \varphi^{4 m}:(3 m) a_{m}\left[\lambda_{4}-(2 m+1) \lambda_{6} \frac{\lambda_{1}}{\lambda_{3}}\right] \\
& \varphi^{2 m}:(2 m) a_{2}\left[\lambda_{4}-(2 m+1) \lambda_{6} \frac{\lambda_{1}}{\lambda_{3}}\right] \\
& -a_{m}\left[\lambda_{6} \frac{\lambda_{2}}{\lambda_{3}}-\lambda_{5}\right] \\
& \varphi^{2 m-1}:(m+1) a_{m}\left[\lambda_{6} \frac{\lambda_{2}}{\lambda_{3}}-\lambda_{5}\right] \\
& \varphi^{0}:-a_{2}\left[\lambda_{6} \frac{\lambda_{2}}{\lambda_{3}}-\lambda_{5}\right] \\
& \varphi^{-1}: a_{2}\left[\lambda_{6} \frac{\lambda_{2}}{\lambda_{3}}-\lambda_{5}\right]
\end{aligned}
$$

From Eqs. (27a), (27b), (27c), (27d), and (27e), we get

$$
\begin{aligned}
B & =\frac{a(t) \lambda(t)-b(t) \gamma(t)}{(2 m-2) \lambda(t) \gamma(t)} \\
A & =-\left[a(t) B^{2}+\gamma(t) B^{3}\right] \\
\frac{q}{p} & =-\left[2 a(t) B+3 \gamma(t) B^{2}\right]
\end{aligned}
$$




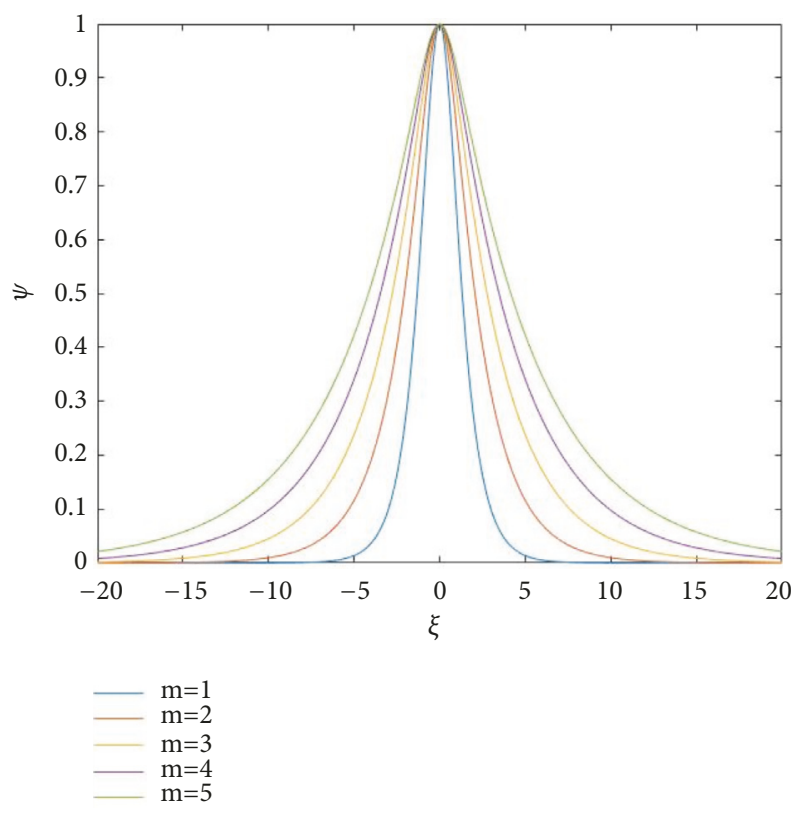

FIGURE 1: Bright soliton waveform of $|\psi|$ for various power indexes $m$.

Next we define $\phi$ as

$$
\phi(\xi)=\varphi^{-1}
$$

So (16) takes the following form:

$$
\frac{d \phi}{\phi^{1-m} \sqrt{a_{m}+a_{2} \phi^{2 m}}}=-d \xi
$$

This equation can be solved with the following analytic solutions:

$$
\varphi=\phi^{-1}=\sqrt[m]{\sqrt{\left|\frac{a_{2}}{a_{m}}\right|} \operatorname{sech}\left(m \sqrt{a_{2}} \xi\right)}
$$

The Modulus of wave function is

$$
|\psi(x, t)|=\sqrt[m]{\sqrt{\frac{a_{2}}{a_{m}} \mid} \operatorname{sech}\left(m \sqrt{a_{2}}(p x+q t)\right)}
$$

Now all of the $a_{2}, a_{m}, A, B$ can be expressed by $a(t), b(t), \alpha(t)$, $\lambda(t), \gamma(t)$ (are constants within the timing range under study), $p$ and $q$, and $q / p$ can be expressed by $a(t), b(t), \alpha(t), \lambda(t), \gamma(t)$.

We can see that the solution (32) obtained by our method is of bright soliton type. The pictorial demonstration of the soliton forms for different power index values $m$ is shown in Figure 1. We see that the NLSE modeling (1+1)dimensional system in power-law dependent media supports bright soliton solution.

\section{Conclusion}

This study investigatied the nonlinear dynamics for the $(1+1)$ dimensional quantum system in the power-law dependent media with the corresponding NLSE model. Using the $F$ expansion method, we derived typical bright soliton solutions under appropriate parameter settings, with key features pictorially demonstrated. Our theoretical treatment showed that a (1+1)-dimensional quantum system with power-law dependent nonlinearity supports bright soliton behavior. The analytical results obtained can be used to guide relevant experimental observation of the soliton dynamics in the (1+1)-dimensional system with power-law dependent nonlinearity.

\section{Data Availability}

Our study is theoretical work consisting of analytical derivation of theoretical model. We do not use previously published data for our work.

\section{Conflicts of Interest}

The authors declare that they have no conflicts of interest.

\section{Acknowledgments}

This work was supported by the National Natural Science Foundation (NSF) of China under Grant nos. 11874185 and 11547024

\section{References}

[1] N. Asif, S. Shwetanshumala, and S. Konar, "Photovoltaic spatial soliton pairs in two-photon photorefractive materials," Physics Letters A, vol. 372, no. 5, pp. 735-740, 2008.

[2] D. D. Ganji, A. Asgari, and Z. Z. Ganji, "Exp-function based solution of nonlinear Radhakrishnan, Kundu and Laskshmanan (RKL) equation," Acta Applicandae Mathematicae, vol. 104, no. 2, pp. 201-209, 2008.

[3] S. Jana and S. Konar, "A new family of Thirring type optical spatial solitons via electromagnetically induced transparency," Physics Letters A, vol. 362, no. (5-6), pp. 435-438, 2007.

[4] R. Kohl, A. Biswas, D. Milovic, and E. Zerrad, "Optical soliton perturbation in a non-Kerr law media," Optics \& Laser Technology, vol. 40, no. 4, pp. 647-662, 2008.

[5] S. Konar, M. Mishra, and S. Jana, "Nonlinear evolution of coshGaussian laser beams and generation of flat top spatial solitons in cubic quintic nonlinear media," Physics Letters A, vol. 5-6, pp. 505-510, 2007.

[6] S. Konar, S. Jana, and W.-P. Hong, "Two-component spatial holographic solitons supported by cross-phase modulation," Physica Scripta, vol. 76, no. 5, p. 470, 2007.

[7] W.-P. Hong, "Optical solitary wave solutions for the higher order nonlinear Schrödinger equation with cubic-quintic nonKerr terms," Optics Communications, vol. 194, no. 1-3, pp. 217223, 2001.

[8] W.-P. Hong, S. Shwetanshumala, and S. Konar, "Modulational instability of optical beams in photovoltaic and photorefractive media due to two-photon photorefractive effect under open circuit condition," Optics Communications, vol. 281, no. 23, pp. 5864-5869, 2008. 
[9] B. Mandal and A. R. Chowdhury, "Spatial soliton scattering in a quasi phase matched quadratic media in presence of cubic nonlinearity," Journal of Electromagnetic Waves and Applications, vol. 21, no. 1, pp. 123-135, 2007.

[10] A. K. Sarma, "Dark soliton switching in an NLDC in the presence of higher-order perturbative effects," Optics \& Laser Technology, vol. 41, no. 3, pp. 247-250, 2009.

[11] S. Shwetanshumala, "Temporal solitons of modified complex Ginzburg Landau equation," Progress in Electromagnetics Research Letters, vol. 3, pp. 17-24, 2008.

[12] A.-M. Wazwaz, "Reliable analysis for nonlinear Schrödinger equations with a cubic nonlinearity and a power law nonlinearity," Mathematical and Computer Modelling, vol. 43, no. 1-2, pp. 178-184, 2006.

[13] A.-M. Wazwaz, "Exact solutions for the fourth order nonlinear Schrodinger equations with cubic and power law nonlinearities," Mathematical and Computer Modelling, vol. 43, no. 7-8, pp. 802-808, 2006.

[14] A. Wazwaz, "A study on linear and nonlinear Schrodinger equations by the variational iteration method," Chaos, Solitons \& Fractals, vol. 37, no. 4, pp. 1136-1142, 2008.

[15] J.-L. Zhang and M.-L. Wang, "Various exact solutions for two special type RKL models," Chaos, Solitons \& Fractals, vol. 37, no. 1, pp. 215-226, 2008.

[16] C. Rogers and W. F. Shadwick, Backlund Transformations and Their Applications, Academic Press, New York, NY, USA, 1982.

[17] S. Li and S.-J. Liao, "An analytic approach to solve multiple solutions of a strongly nonlinear problem," Applied Mathematics and Computation, vol. 169, no. 2, pp. 854-865, 2005.

[18] Y. Wang and Y. Zhou, "Exact soliton solutions of the generalized Gross-Pitaevskii equation based on expansion method," AIP Advances, vol. 4, Article ID 067131, 2014.

[19] S. Li, Y. Wang, Y. Zhou, J. Guo, G. Gao, and Y. Zhang, "Soliton dynamics for one dimensional quantum system incorporating higher-order dispersion effect and nonlinear interactions," Chinese Journal of Physics, vol. 55, no. 6, pp. 2436-2440, 2017.

[20] M. Saha, A. K. Sarma, and A. Biswas, "Dark optical solitons in power law media with time-dependent coefficients," Physics Letters A, vol. 373, no. 48, pp. 4438-4441, 2009. 


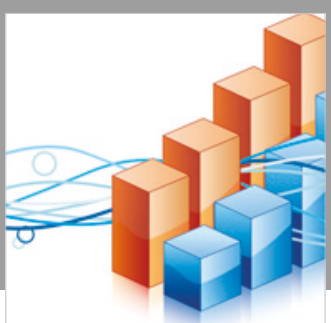

Advances in

Operations Research

\section{-n-m}
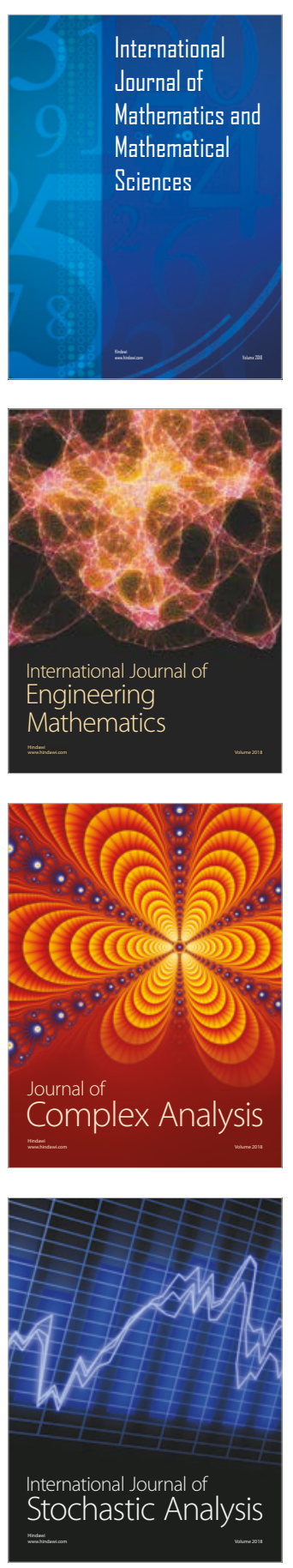
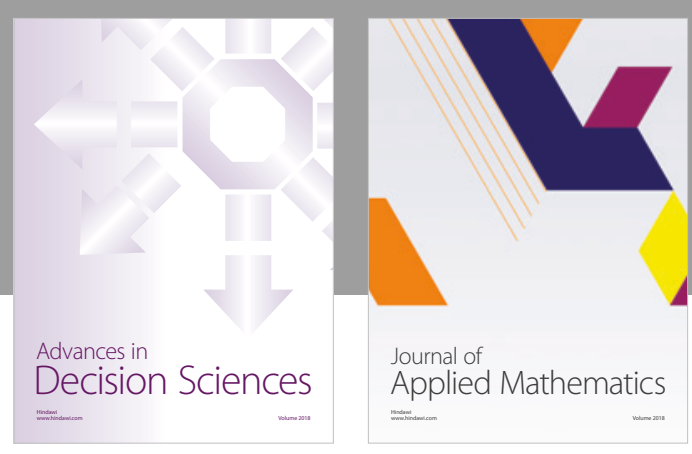

Journal of

Applied Mathematics
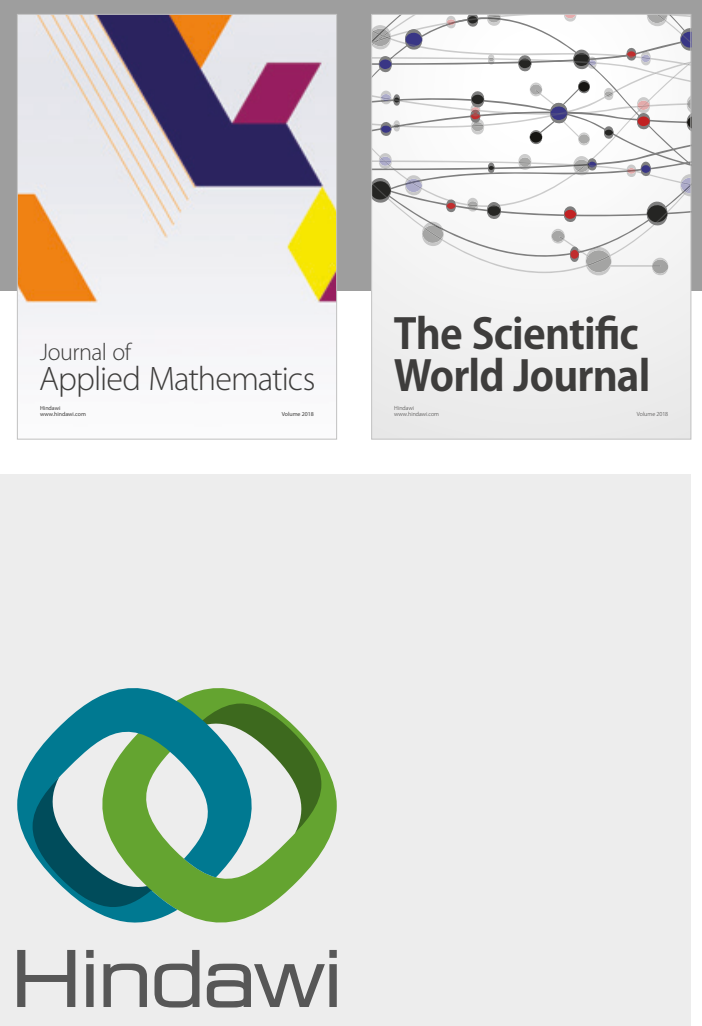

Submit your manuscripts at

www.hindawi.com

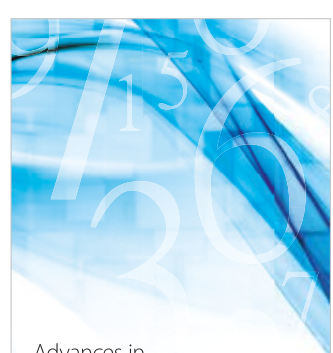

Advances in
Numerical Analysis
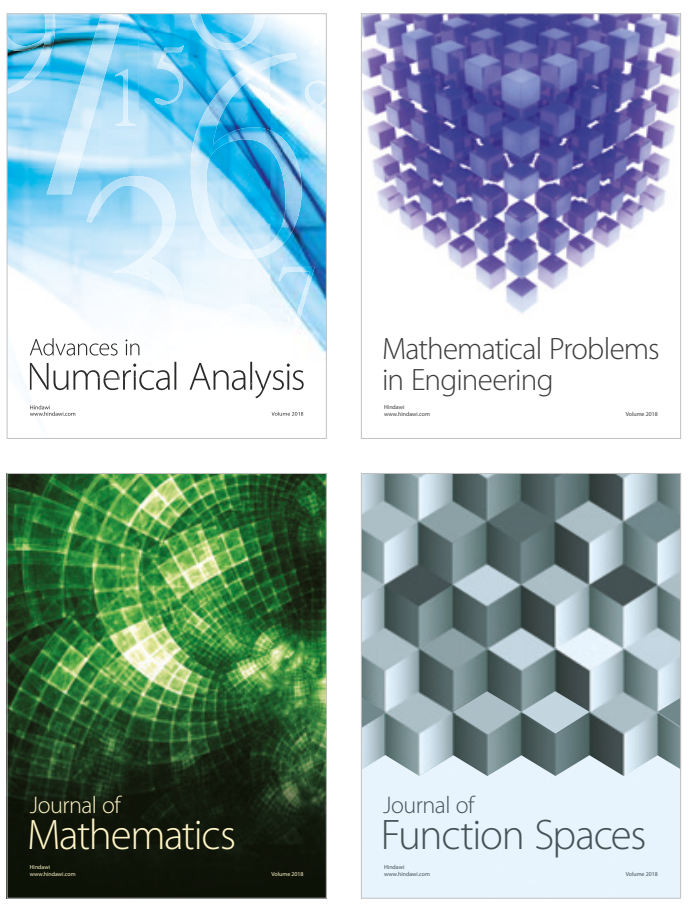

Mathematical Problems in Engineering

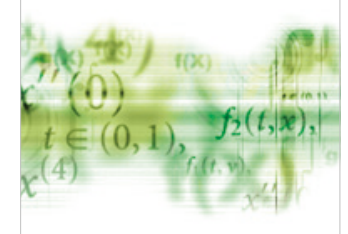

International Journal of

Differential Equations

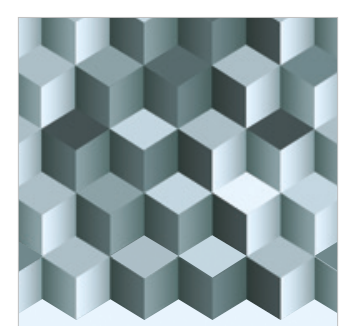

Journal of

Function Spaces

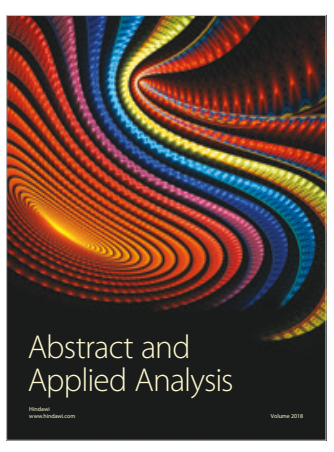

The Scientific

World Journal

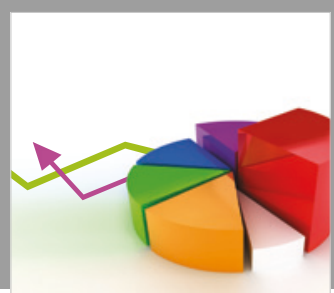

Journal of

Probability and Statistics
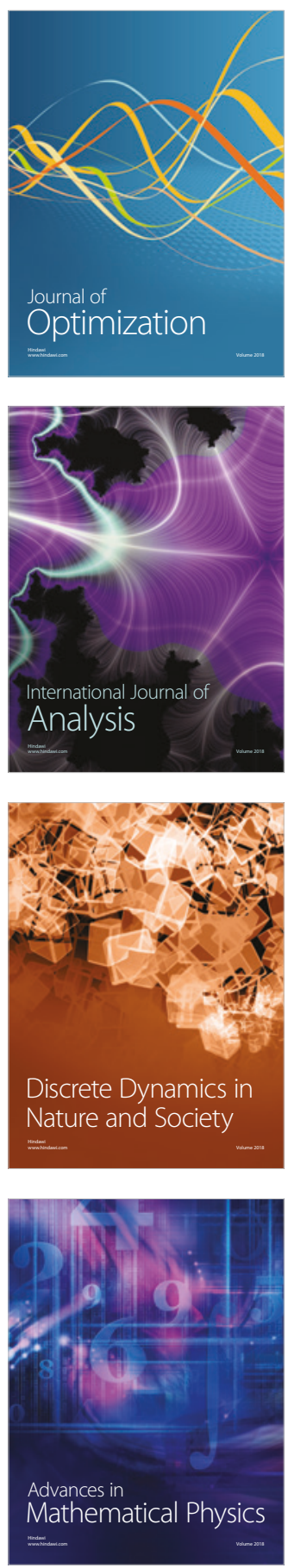